\title{
THE EFFECT OF THE CONTROL SYSTEM CREATION PROCESS
}

\author{
Venelin Terziev ${ }^{1}$ and Evgeniy Stoyanov ${ }^{2}$ \\ ${ }^{1}$ Professor, Ph.D., D.Sc. (National Security), D.Sc. (Ec.), University of Rousse, Rousse, Bulgaria; \\ National Military University, Veliko Tarnovo, Bulgaria; University of Telecommunications and Post, \\ Sofia, Bulgaria, terziev@skmat.com \\ ${ }^{2}$ Professor, Ph.D., D.Sc., National Military University, Veliko Tarnovo, Bulgaria
}

\begin{abstract}
The expose details the specifics of the act of creating a control system in the modern organization as an essential moment for organizational development and success. For this reason, the whole technology is presented in stages in which emphasis is placed on the leading principles justifying the stage content. The final stage is properly considered, as at that point the newly created control system becomes the object of control or territory for manifestation of self-control. The aim of the study is to provide evidence that a welldesigned control system is a key factor for organizational success. The tasks resulting from the decomposition of the goal are involved in revealing the principle beginning in the content of each stage, the tracking of the conceptual continuity between the elements of the presented technology and the definition of concrete solutions accompanying the process development and problems.
\end{abstract}

Keywords: control, system, principle, self-control, technology.

\section{MAIN TEXT}

In the current reality, social phenomena are becoming more and more. Leading analysts and philosophers reveal that this is the result of the poor functioning of the basic social systems, such as social, political and economic. The growing imbalance between them proves that key social mechanisms fail or simply do not work in sync. One of these key factors is control. It is strange that control is being spoken more and more often. The paradox is that its deficit is becoming more and more serious. The very idea has proven its sustainability, but it is becoming increasingly neglected in today's organizations and society itself. Analyses of the failure or failures of modern organizations, regardless of their subject of business, are increasingly proving that one of the reasons is either the delay in the creation of an adequate control system or the low efficiency of the control activity. Reasons create the conditions to consider that although the essential elements (subject, object standards, and toolbox) are available, they do not interact with each other in the required synchronization.

The process of creating or designing the control system is linked both to registered links and analysed relationships between the elements mentioned, as well as to a specific design technology that is logically 
organized and, at the same time, subject to principles guaranteeing the solidity and balance of the control system created (1997).

The initial stage of the designing technology of the control system is concerned with the choice of standards. Its implementation is based on the results of the activities to assess the planned and expected state of the controllable system. The specific choice of the subject and the significance of the standards is a function of the realized need to control and analyse the influence of the controllable parameters on the achievement of the planned state. This stage is characterized by the application of several principles that are decisive for its successful completion.

The guiding and basic principle is "the principle of purposefulness" or the achievement of full compliance and coherence between planned objectives and tasks and the chosen standards. Its implementation is a mechanism that forms the hierarchy of standards, ranking them by significance according to the plan implementation. Compliance with standards is unambiguous and necessary for the success of the entire initiated process.

„Reasonable sufficiency” is the second principle according to which only controllable parameters that are decisive for deviations from the planned state can be defined as a subject of control. The application of this principle has a preventive effect in terms of "overdose" of control impact, increasing dissatisfaction caused by control and limitation of initiative.

The third principle of the "decision-making unit” or its differentiation is manifested after an analysis of relationships and connections in the sub-control system, by the standards of the most sensitive and decisive parameters that imply conditions and causes for deviant behaviour.

The proper observance of the stated principles relies on the process of designing the control system and the development of basic essential characteristics of the control activity, such as focus on results, economy, democracy, etc. (1997). The development of this trend is observed within the entire design process, systematically and consistently. The very systemic nature of the process does not allow its occasional or incidental manifestations.

The second stage in the design of the control system encompasses the analysis of control relationships that predict deviations from standards. Based on a critical analysis of stakeholders' motivation and potential for compliance with the standards, the status of actual control relationships is established. The control influence on the behaviour of personality formations that do not comply with the established standards is increased purposefully.

In the next phase, at this stage, possible options for restructuring and optimization of the control relations according to the standards are determined. After an analysis of the results achieved, the potential control objects are identified initially and the requirements for the incentive system are formulated. As a result, for each standard, an overview of the control objects, the possible subjects and the measures that guarantee the selected subjective position is formed.

In the implementation of the second stage it is necessary to take into account the fact that then the object structure of the control system is defined more specifically. With regard to the subject structure, things are still quite unidentified, as the possible subjects are in informal relation to each other as well as to the controllable parameters. Similar is the state of the incentives' system by the instrument structure. Again, there are options for incentives that are not organized in a particular structure, but are independent, initially involved groups around individual controllable parameters.

In evaluating the results of the second stage, it is also worth taking into account the fact that the differentiation of the subject structure and the incentive system is based on the reasonability and commitment of authority, based on the analysis of the possibility of applying the control impact in its regulatory aspect. Among the principles to which the process is desirable to adhere at this stage is the principle of bringing the subject the closest to the object of control. Proximity provides optimal opportunities for observation and analysis. With regards to the subject, it can be said that there is a specific form of diversification of the control relations and, in particular, the imposition of the qualities of a subject and object of control to certain persons or groups simultaneously viewed in different situations.

In the course of this stage, the principles of control activity mentioned in the previous stage have created the impression of complete presence and manifestations. Here, it is only right to think and take into account the impact of the principle of economy.

The next (third) stage in the design is to analyze the forms and methods of control. The starting point for its implementation is the previous two stages. On the basis of the built structure of the standards and the 
characteristics of the parameters, which are the subject of its influence, the choices of the optimal forms, methods and technology for implementation of the control activity are applied. This choice is the basis on which a larger view of the instrumental structure of the control system is built.

A significant difference from the content of the previous stages is the presence and reporting not only of the power resources but also the increase of the weight of the information and analytical aspects in the functioning of the control system (2010).

The greatest importance at this stage is the emergence of the principle of compliance of control with controlled activities. Its application predetermines the expansion of the influence of effective control activity towards performance, flexibility, economy and prevention. It is also essential for this stage to take into account the principle of clarity and comprehensibility (transparency) of the selected forms, methods and control instruments. They should ensure the possibility of maximally nearing the expanded subject structure to the control object.

In the fourth stage of the process of creating a control system, the concepts of the subjective and instrumental structure of the control system are combined, assessing their conformity with the object structure and the system of standards. The subjective structure in the stage is a peculiar epicenter of logical and constructive activity because it provides a balance in the future effective operation of the control system. In this sense, adjustments are a limiting factor for optimal deployment of other structures. It is for this reason that it is essential to find the capabilities the subjective structure to be further developed at different hierarchical levels. Of particular importance in the design of these levels is the application and compliance with the principle of exhaustion of the bottom-up possibilities, which optimizes the use of the principle of maximal nearing of the subject to the control object.

The application of the said set of consolidated principles creates prerequisites for defining the functions and tasks of the control subjects in an operational, tactical and strategic aspect. It creates conditions with the majority of the operational-tactical control activity to engage lower and middle hierarchical levels. This enables the higher-level controllers to concentrate their strategic control efforts on organizing a strategic orientation of control activity.

As a result of the draft structure for the subject structure (2012), it is now possible to speak more of a certain range of variants of instrumental structure and on its basis to refine the object structure and structure of standards. In general, at the end of this stage, a certain clarity and relatively complex vision is created for the structure of the entire control system in its distinctive specificity and reasonable interdependence.

The subject of the fifth final stage of the process is the remaining open question for the control system control subjects. In other words, the structuring of specialized bodies for the management of the control system is related to the real status and capabilities of the general management bodies in the control activities, the functions of strategy, planning, organization, motivation and control.

In the near future there are several significant problems in the management of the control system, the decision of which is the prerogative of the managing authority.

The first problem is related to the design of the control system itself and to the analyzes carried out in it, which justify the research itself.

The second problem arises from the mandatory condition for the success of the process of operating a control system, i.e. the establishment of methodological unity of control activity. This includes not only the development of instrumentation and control technology, but also the creation of conditions for its effective application.

The third problem is related to the constant coordination of the control activity and the continuous refinement of the control system. As far as this is inevitable, this type of fine tuning is a type of adaptation to emerging circumstances.

The fourth problem stems from the multifunctional functionality of the control system in particular and as information. The heterogeneous character of the information predetermines the differentiation of a narrow specialization in terms of the overall application of the information used in the three established areas: financial-accounting, statistical and socio-psychological.

The fifth problem is related to ensuring high reliability of the control system by designing and controlling the control by the authorities controlling the control system. The solution to this problem has a variable success, which is related to changes in economic conditions and changes in the behavior of economic systems. 
The analysis and evaluation of problematic events reflects the content at this stage. It evaluates the designed control system from the point of view of its economy (2007). The main task at the stage is to assess whether management is able to pay the expected cost to the control system and where possible the areas for cost effective optimization.

A useful means of identifying excessive and unnecessary costs, which can increase the economy of the control system without affecting its performance, is a functional-value analysis (1988). The technology is realized in a multi-stage process.

Stage I. A structural-component model of the analyzed control system is being prepared. This model reflects the composition and the co-subordination of the components according to their functional engagement.

Stage II. A functional model of the control system is created, in which the specifics of the components of the system are defined and formulated functionally.

1.Main function - an external feature that defines its purpose, nature and purpose;

2.Secondary functions - external component functions, reflecting additional beneficial effects consistent with the objectives of the control system;

4.Auxiliary functions - these are internal functions that contribute to the realization of the basic ones.

Stage III. A functional-structural model of the control system is made in which the system components are linked to the functions they perform.

Stage IV. A functional-value diagram is produced, reflecting the ratios between the metricized significance of the individual functions and the costs incurred by them through the various components of the control system. The significance of the individual functions is formed on the basis of the objectives, tasks and requirements of the control system.

Through the application of the functional-value analysis can be specified:

- the necessary and useful functions of the control system components that meet its objectives and requirements;

- the unnecessary and useless features of components that do not contradict its objectives and requirements, but unnecessarily exceed some of its parameters and make it unnecessarily expensive;

- unnecessary and harmful functions of the control system components that create a high-conflict environment based on objectives, tasks and value.

It is necessary to note that the application of this format of analytical activity is compatible only in the conditions of normal development and functioning of the control system when thinking and looking for a model for optimization of its functionality but also efficiency (2005).

In periods of crisis development, the principle of economy is most adequately implemented through budgetbased zero-level planning. In applying this optimization model, the economic factor takes a central lead on all the results achieved by the individual stages. The effect of the budget planning of the control system on a zero basis provokes the conclusion that complex revisions and revision of the operating costs of each control system are necessary.

The written statement provides sufficient grounds for making some conclusions:

First. The process of building a working control system is a complex and creative process that is becoming more and more important for the effective work of any modern organization.

Second. Finding optimal synergy between the control system's performance and the work of the organization itself becomes one of the few alternatives that undoubtedly create the conditions for stable and successful development.

Third. If the presence of control in any system is a rightful presence justified by the unity of the management process, in the implementation of control in the control system itself, there can also be talked about searching for hidden capabilities, and at the highest results and operations and processes to be optimized in order to achieve maximum effect.

Fourth. The disclosure in the content of each stage of the technology for the creation of control systems of the tangible presence of separate control principles proves that control is a structured, institutionalized, socially relevant activity based on theoretical and philosophical developments and closely related to socioeconomic development. The existence of the principle beginning proves that this form of critical social 
attitude has past and present, and the attitude towards the registered results will indicate what its future will be.

In conclusion, it can be said that as a theoretical development, science and control concepts are in a very serious and advanced phase. The problem that society has to solve is how to consolidate theory and practice in the best way for all stakeholders and how to effectively control all levels, including the behavior of high-ranking institutions in the social hierarchy.

\section{REFERENCES LIST}

Reference by Functional Standing Analysis, Order. Carpunina M., B. Maidanchika, M., (1988).

Simeonov O. (1997), Theoretical Control Issues, Academic Consult, C.

Stoyanov E. (2005), One Successful Idea in Strategic Control, Anthey-TN, Bourgas.

Kaplan R., P. Couper, (2007) Functional Analysis, M., Villiams.

Stoyanov E., (2010), Financial Control, Libra Scorpio, B.

Stoyanov E, (2012), Theory of Financial and Economic Analysis, Libra Scorpio, Burgas. 